\title{
Osteoporosis diagnostics in patients with rheumatoid arthritis
}

\author{
Małgorzata Węgierska ${ }^{1}$, Marta Dura², Einat Blumfield ${ }^{3}$, Paweł Żuchowski ${ }^{4}$, Marzena Waszczak \\ Stawomir Jeka ${ }^{1}$ \\ ${ }^{1}$ Clinic of Rheumatology and Systemic Connective Tissue Disorders, J. Biziel University Hospital No. 2, Bydgoszcz, Ludwik Rydygier \\ Collegium Medicum in Bydgoszcz, UMK in Torun, Poland \\ ${ }^{2}$ Department of Radiology, J. Biziel University Hospital No. 2, Bydgoszcz, Poland \\ ${ }^{3}$ Jacobi Medical Center, Albert Einstein College of Medicine, Bronx, NY, USA \\ ${ }^{4}$ Independent Section of Scientific Research Coordination, J. Biziel University Hospital No. 2, Bydgoszcz, Poland \\ ${ }^{5}$ Centre for Clinical Trials, Warsaw, Poland
}

\begin{abstract}
Rheumatoid arthritis (RA) is a chronic systemic connective tissue disease. The development of comorbidities often occurs in the course of RA. One of them is osteoporosis, which has serious social and economic effects and may contribute to the increase in the degree of disability and premature death of the patient. Due to the young age in which RA disease occurs, densitometry (DXA) of the lumbar spine is the basic examination in osteoporosis diagnostics. In the course of RA, much more frequently than in healthy persons of the same age, osteoporotic fractures of vertebral bodies occur, which hinder a correct assessment in the DXA test. Rheumatoid arthritis patients often undergo computed tomography (CT) examination of the abdominal cavity for other medical indications than suspected spinal injury. Then, CT examination may also serve for the assessment of bone density, especially in patients with osteoporotic fractures.
\end{abstract}

Key words: bone densitometry, quantitative computed tomography, osteoporosis, rheumatoid arthritis.

\section{Introduction}

Rheumatoid arthritis (RA) is an immune-mediated inflammatory connective tissue disease. Symmetrical inflammation in the joints of hands and feet is a characteristic feature of RA. In the course of the disease, very often the internal organs are affected and many complications occur, including the development of co-morbidities such as secondary amyloidosis, diabetes mellitus, cardiovascular diseases or osteoporosis.

Osteoporosis occurs very often in the course of RA, which requires particular attention during patients' treatment.

\section{Osteoporosis}

Osteoporosis is one of the most common diseases of the skeletal system [1]. In its course, not only reduced bone density occurs, but also structural changes appear, which is why the bones become more brittle. This is associated with a significant increase of the risk of fracture.

It is estimated that approximately $50 \%$ of women and $20 \%$ of men will suffer from a fracture due to osteoporosis [2]. In 2000, in Europe, 620,000 femoral neck fractures occurred in patients suffering from osteoporosis $[3,4]$. Compression spinal fractures constituted a similar number of fractures in the same year $[3,4]$.

Osteoporotic fractures are closely associated with an increase in mortality. Approximately $20-30 \%$ of patients die as a result of long-term immobilisation and postoperative complications within 6 months after the incident [5]. Femoral neck fractures cause ca. 50\% of deaths in this groups. Spinal fractures come second, and they are responsible for ca. $28 \%$ of deaths [4].

Address for correspondence:

Małgorzata Węgierska, Clinic of Rheumatology and Systemic Connective Tissue Disorders, J. Biziel University Hospital No. 2, Ujejskiego 75, 85-168 Bydgoszcz, Poland, e-mail: nargita@wp.pl

Submitted: 16.02.2016, Accepted: 25.02.2016 


\section{Osteoporosis in patients with rheumatoid arthritis}

The frequency of occurrence of osteoporosis in patients with rheumatoid diseases, including RA, is about $50 \%$ [6]. The course of osteoporosis is closely connected with the activity of the underlying disease and other risk factors, e.g. age, low BMI (<18), early menopause (<45), low-energy fractures, renal failure, diabetes, smoking, alcohol use, high bone turnover, vitamin D deficiency, low intake or impaired absorption of calcium and low calcium serum concentration. Also active inflammation and glucocorticoid therapy, long disease duration, malabsorption and the deficiency of sex hormones are recognized risk factors for osteoporosis [6].

Reduced bone density within joints, called periarticular osteoporosis, is the first and most common radiological lesion, that is stage I according to Steinbrocker in the course of RA. It is associated with bone erosion and joint space narrowing. Moreover, in the course of RA very often secondary osteoporosis occurs due to the use of glucocorticosteroid (GC) therapy. Currently, it is said that the mechanisms causing periarticular and secondary osteoporosis are at least partly the same [7].

Development of osteoporosis in patients with RA leads to further decline of the quality of life and increase in the costs of treatment and rehabilitation. The frequency of occurrence of femoral neck fractures and vertebral compression fractures in patients with RA is twice as high as in healthy persons of the same age [8].

The risk of osteoporotic fractures is closely associated with the disease duration as well as with other factors such as low body mass index, difficulties with movement, GC therapy and sarcopenia.

\section{Osteoporosis diagnostics}

Currently, a dual energy X-ray absorptiometry (DXA) test performed from the lumbar spine or femoral neck is the gold standard in osteoporosis diagnostics. The result is expressed as a standard deviation from the peak bone mineral density (BMD), called the T-score. The standard deviation from the mean BMD value for a given age is called the Z-score.

According to the recommendations of the International Society for Clinical Densitometry (ISCD), the T-score is used in the case of men aged 50 or more and women after menopause. Then, the Z-score is used in the case of men below 50 years of age and women before menopause.

BMD assessment in the DXA test should be performed first based on the lumbar spine examination in persons below 65 years of age [9].
The DXA test measures BMD only, which is sometimes a very serious limitation. Test results in no way include the bone structure, which is equally important in the assessment of bone resistance to fracture.

Unfortunately, in the case of occurrence of degenerative lesions in an examined area, very often a BMD increase occurs, which in turn may lead to masking of osteoporosis in DXA tests and thus, underestimation of the risk of occurrence of fractures [10, 11].

In extreme cases, even in compression fractures of vertebrae as a result of osteoporosis, BMD values may correspond to the normal bone density or osteopenia, which of course is inconsistent with the clinical condition of the patient.

Such a situation is presented in Figure 1. A patient suffering from RA for 11 years had a DXA test. The DXA test was performed due to the suspected osteoporosis in physical examination - loss of height, stooped posture and reduction of distance between the ribs and pelvis.

\section{Osteoporosis diagnostics in patients with rheumatoid arthritis}

Rheumatoid arthritis most often affects young people at the age of 30-50 years. In the case of diagnostics for osteoporosis in this age group in accordance with ISCD recommendations, the examination performed should be the DXA test of the lumbar spine.

The greatest bone turnover occurs in the spine due to a vast predominance of cancellous bone, which favours the examination of this area in particular. Owing to this, it is possible to quickly diagnose osteoporosis as well as to track the changes occurring during the treatment.

In BMD assessment major lesions should not be expected in the femoral neck due to the relatively young age of RA patients. BMD loss in the area of the femoral neck starts only around the age of 65 [9].

In the case of RA patients, it is not recommended to perform BMD assessment based on the examination of the distal end of the radius. Due to RA and the inflammatory process connected with it, causing a local BMD loss, this examination will be characterised by over-detection of osteoporosis [7].

Due to the fact that the fractures of vertebral bodies are very often painless and are usually not diagnosed for a long time, also examination of the lumbar spine is advocated; only about 30\% of these kinds of fractures present pain symptoms. Correct diagnosis is often hindered by wrong identification of the pain in the thoracic and lumbar spine as the inflammation of nerve roots [3].

Due to this, during physical examination it is necessary to take into consideration the loss of height and 
increased thoracic kyphosis, which is connected with leaning the head forward or reducing the distance between the costal arches and iliac crest of the pelvis.

The above lesions may suggest that there was a compression fracture of the vertebral body in an examined patient. Detection of a vertebral body compression fracture is extremely important from the point of view of further treatment. In persons who have suffered from one such fracture, the risk of subsequent fractures increases from 5- to 7-fold [12].

Numerous examinations performed confirms a significant increase of the risk of fractures in RA patients as compared to healthy persons of the same age $[8,13]$. In a study performed on a group of 191 female RA patients after menopause and a control group of 713 female patients after menopause (in both groups, patients were within the 45-65 age group), the assessment of the number of compression fractures was performed based on X-ray images [13]. It was found that in the RA patient group compression fractures were twice as frequent as in the control group [13].

These types of examinations confirm how important the lumbar spine area in BMD assessment is. Unfortunately, DXA tests are sensitive to degenerative lesions and compression fractures in the lumbar spine $[10,11]$.
The patient whose DXA test result is presented in Figure 1 also underwent CT of the abdominal cavity. Due to clinical symptoms indicative of osteoporosis and risk factors of its development (long-term GC therapy and smoking), the radiologist additionally performed assessment of the lumbar spine. It was found that the patient had a low-energy compression fracture in L3 - a fracture characteristic of osteoporosis, which is presented in Figure 2.

The case presented in Figures 1 and 2 illustrates the problems in the osteoporosis diagnostics characteristic for RA patients. Hence, using other methods in osteoporosis diagnostics seems to be more effective.

\section{Quantitative computed tomography}

One of the alternative methods for BMD assessment is quantitative computed tomography (QCT). QCT is a far more precise BMD measurement method than the DXA test [14]. One of the advantages of this examination is the possibility to measure volumetric bone mineral density (VBMD), which allows one to avoid certain errors in measurements that are connected with surface BMD assessment in the DXA test.

Undoubtedly, the greatest advantage of QCT is the possibility of imaging of bone structure and performing
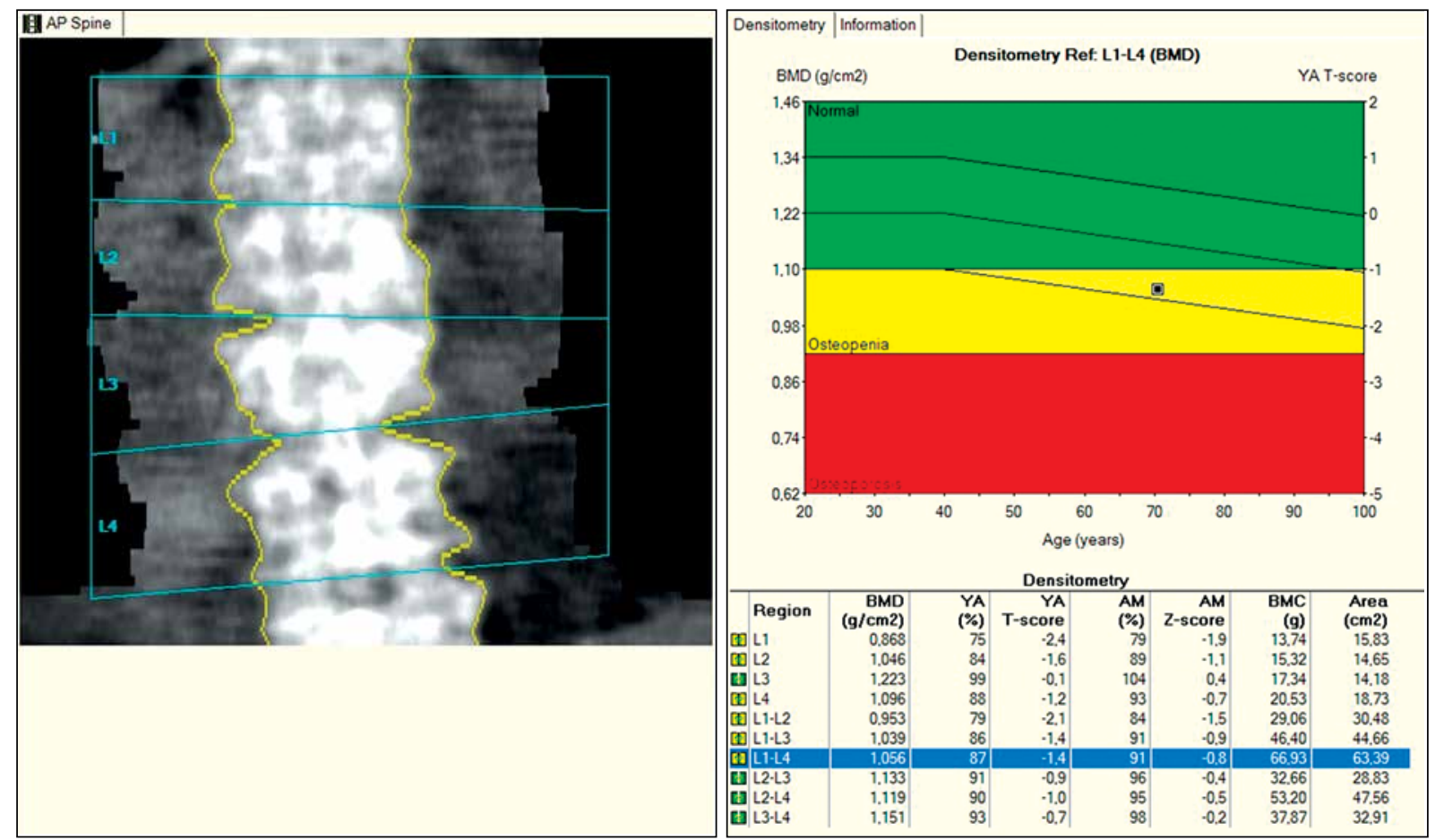

Fig. 1. DXA test result. Suspected degenerative lesions in L3 vertebra (significant difference in the value of the T-score in comparison to other vertebrae). In accordance with ISCD recommendations, the L1-L2 section was used for the assessment of bone density. Based on the DXA test, osteopenia was diagnosed. 

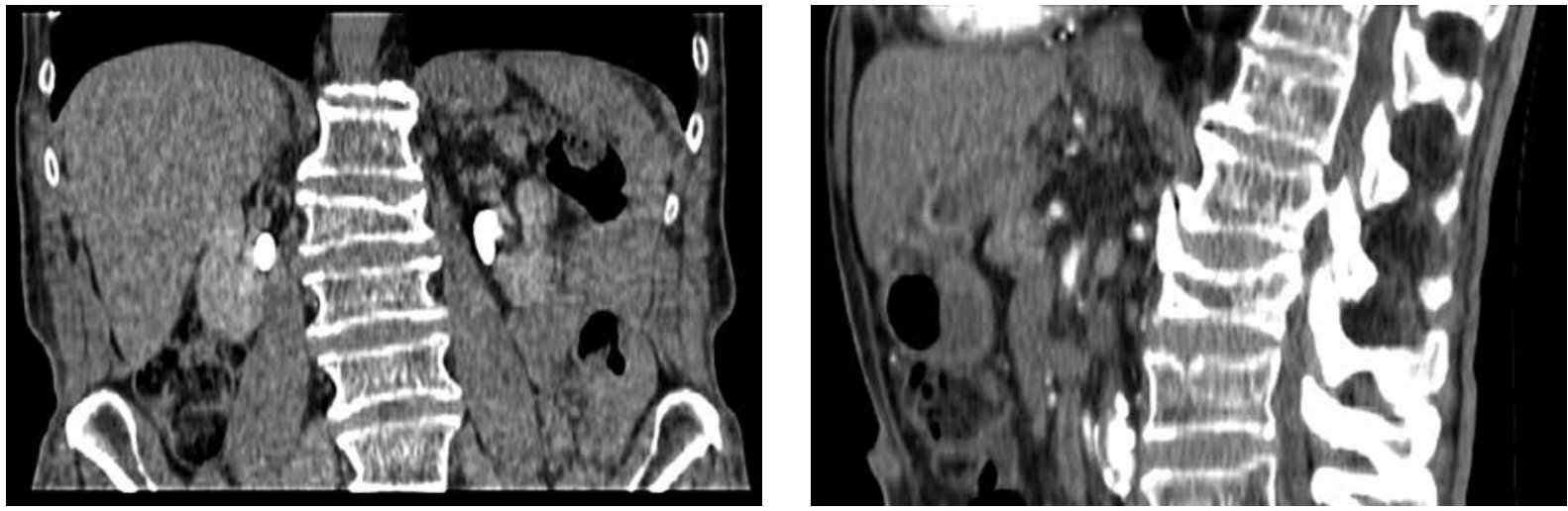

Fig. 2. Abdominal cavity CT examination - visible, low-energy fracture of the $L 3$ vertebral body. Loss of height of $L 3$ vertebral body with a fracture of the superior vertebral endplate. Grade 2 according to Genant's classification.

the differentiation between the cortical and trabecular bone $[15,16]$. Owing to this, it is possible to perform VBMD assessment in the lumbar spine, even in patients with major degenerative lesions in this area.

In the QCT examination, VBMD assessment may be based only on trabecular bone, whose bone turnover is much faster than in cortical bone [17]. Apart from that, most degenerative lesions refer to cortical bone.

The lack of possibility of such differentiation in the DXA test causes both the masking of osteoporosis due to BMD increase caused by degenerative lesions, and the necessity to maintain a longer time interval between the examinations to demonstrate the changes in BMD values.

Unfortunately, a major limitation of the number of performed QCT examinations is the relatively large dose of $x$-ray radiation to which the patient is exposed, and lack of standardisation of examinations between the CT devices from different manufacturers. The radiation dose in the QCT examination when using appropriate protocols is 2,500-3,000 $\mu \mathrm{Sv}$, and in the DXA test it is approximately $13 \mu \mathrm{Sv}$ [18].

The most common method of $\mathrm{VBMD}$ assessment in QCT requires the use of a phantom, which causes the need to plan this kind of examination sufficiently in advance. Techniques enabling the VBMD assessment based on standard phantom-less CT examination of the abdominal cavity are less common [19, 20].

However, despite these drawbacks, in recent years QCT examinations are being performed more often [21]. In 2013, the American College of Radiology (ACR) updated the guidelines for QCT examinations and introduced the definition of osteopenia and osteoporosis based on VBMD measurement [22]. Bone density of more than $120 \mathrm{mg} / \mathrm{cm}^{3}$ corresponds to the correct bone density. It is assumed that bone density within the range of 80-120 $\mathrm{mg} / \mathrm{cm}^{3}$ indicates osteopenia, and bone density below $80 \mathrm{mg} / \mathrm{cm}^{3}$ indicates osteoporosis.
Due to the high quality of the obtained image, it is possible to easily detect fractures in the QCT examination which in DXA tests are invisible, as shown in Figure 3. Based on the QCT examination, it is also possible to differentiate vertebral fractures which occurred as a result of major injuries from low-energy fractures associated with osteoporosis [23].

The future of screening in osteoporosis diagnostics may belong to phantom-less QCT examinations, in which the bone density is determined based on the density of the surrounding soft tissues (Fig. 3).

A steady increase in the number of performed CT examinations, including abdominal cavity CT examinations, has been observed for many years. These examinations may be used for VBMD assessment of the lumbar spine, and they are also applicable to the population that should undergo an examination for osteoporosis.

Further development of programs like Radnostics (Fig. 3) should to a large extent remove the inconveniences associated with standard QCT examinations, i.e. special planning of the examination and additional effort connected with vBMD assessment. With the constantly growing number of performed CT examinations, this diagnostic method may become one of the basic screening methods.

\section{Summary}

Considering the fact that RA patients, due to various complications in the course of the underlying disease, undergo CT examinations more often than healthy persons of the same age, QCT, especially phantom-less, may constitute a good alternative method of bone density assessment as compared to DXA tests.

The relatively young age of RA patients, increased risk of osteoporosis occurrence and twice as high number of compression fractures in comparison with healthy 


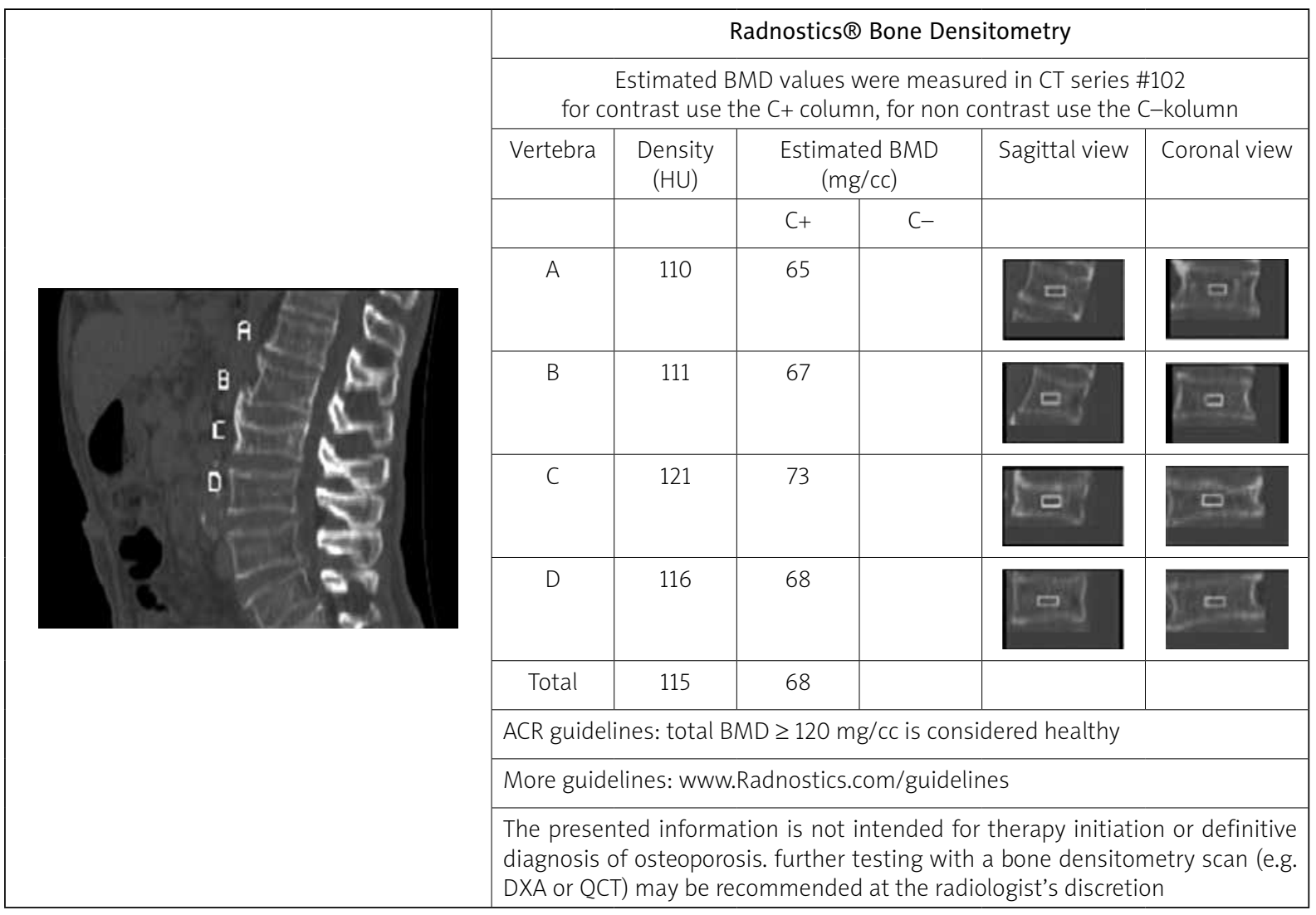

Fig. 3. QCT examination. VBMD assessment was performed based on phantom-less CT examination of abdominal cavity using the fully automatic Radnostics computer program (Radnostics LLC). The image shows the fracture of the L3 vertebral body; in accordance with the ACR criteria osteoporosis was diagnosed, which conforms to the clinical picture.

persons also favour the use of the QCT examination. Moreover, limitations connected with the DXA test occur in patients with exacerbated degenerative lesions in the spine.

Certainly, modern DXA devices enable the performance of morphometry, which should serve to identify fractures of the vertebral bodies [6]. However, the quality of the imaging is much lower than in the case of the QCT examination, and it requires different positioning of the patients than in the regular DXA test for BMD assessment, which is connected with the performance of an additional examination. This requires more time than the time needed for performance of the QCT examination and assessment of potential fractures of vertebral bodies using an automatic computer program such as Radnostics.

Taking into consideration the fact that VBMD assessment in CT examination may be performed automatically and it does not interfere with the standard protocol of the $\mathrm{CT}$ examination of the abdominal cavity, after the evaluation of advantages and disadvantages of the DXA test and QCT examination, it may be assumed that in RA patients the QCT examination may have a significant impact on the therapeutic procedure. In many cases, it will enable faster detection of BMD reduction than the DXA test. This refers mainly to the lumbar spine examination.

It needs to be noted that the low detectability of fractures in CT examinations is connected with the fact that these examinations are performed for other diagnostic reasons than the search for fractures. Radiologists rarely use the Genant classification for the assessment of fractures; thus, they usually only describe major fractures which correspond to grade 3 fractures according to the Genant classification [6]. In the QCT examination result, in which only vertebral bodies are visible, it is possible to easily diagnose minor fractures of vertebral bodies.

For the abovementioned reasons, the QCT examination should be used as one of the examinations in the diagnostics of osteoporosis in RA patients who undergo $\mathrm{CT}$ examinations of the abdominal cavity for other medical reasons.

The authors declare no conflict of interest. 


\section{References}

1. Tkaczuk-Włach J, Sobstyl M, Jakiel G. Osteoporoza - obraz kliniczny, czynniki ryzyka i diagnostyka. Prz Menopauzalny 2010; 2: 113-117.

2. Pickhardt PJ, Lee LJ, del Rio AM, et al. Simultaneous screening for osteoporosis at CT colonography: bone mineral density assessment using MDCT attenuation techniques compared with the DXA reference standard. J Bone Miner Res 2011; 26: 2194-2203.

3. NAMS continuing medical education activity. Management of osteoporosis in postmenopausal women: 2010 position statement of The North American Menopause Society. Menopause 2010; 17: 23-56.

4. Kanis JA, McCloskey EV, Johanson $\mathrm{H}$, et al. European guidance for the diagnosis and management of the osteoporosis in postmenopausal women. Osteoporos Int 2013; 24: 23-57.

5. Kanis JA, Oden A, Johnell O, et al. The components of excess mortality after hip fracture. Bone 2003; 32: 468-473.

6. Leszczyński P, Hrycaj P, Mackiewicz S. Osteoporoza u chorych na reumatoidalne zapalenie stawów - realny problem czy fikcja? Ocena występowania złamań trzonów kręgowych u kobiet $z$ reumatoidalnym zapaleniem stawów $w$ wieku do 50 lat. Reumatologia 2007; 45: 362-368.

7. Vis M, Havaardsholm EA, Haugeberg G, et al. Evaluation of bone mineral density, bone metabolism, osteoprotegerin and receptor activator of the NFKB ligand serum levels during treatment with infliximab in patients with rheumatoid arthritis. Ann Rheum Dis 2006; 65: 1495-1499.

8. Hoes JN, Bultink IE, Lems WF. Management of osteoporosis in rheumatoid arthritis patients. Expert Opin Pharmacother 2015; 16: 559-571.

9. Schneider DL, Bettencourt R, Barrett-Connor E. Clinical Utility of Spine Bone Density in Elderly Women. J Clin Densitom 2006; 9: 255-260.

10. Rand T, Seidl G, Kainberger F, et al. Impact of spinal degenerative changes on the evaluation of bone mineral density with dual energy X-ray absorptiometry (DXA). Calcif Tissue Int 1997; 60: 430-433.

11. Tenne M, McGuigan F, Besjakov J, et al. Degenerative changes at the lumbar spine-implications for bone mineral density measurement in elderly women. Osteoporos Int 2013; 24: 1419-1428.

12. Klotzbuecher CM, Ros PD, Landsman PB, et al. Patients with prior fractures have an increased risk of future fractures: a summary of the literature and statistical synthesis. J Bone Miner Res 2000; 15: 721-739.

13. Spector TD, Hall GM, McCloskey EV, et al. Risk of vertebral fracture in women with rheumatoid arthritis. BMJ 1993; 306 (6877): 558.

14. Li N, Li XM, Xu L, et al. Comparison of QCT and DXA: Osteoporosis Detection Rates in Postmenopausal Women. Int J Endocrinol 2013; 2013: 895474.

15. Ito M, Hayashi K, Kawahara Y, et al. The relationship of trabecular and cortical bone mineral density to spinal fractures. Invest Radiol 1993; 28: 573-580.
16. Genant HK, Block JE, Steiger P, et al. Quantitative computed tomography in assessment of osteoporosis. Semin Nucl Med 1987; 17: 316-333.

17. Adams JE. Quantitative computed tomography. Eur J Radiol 2009; 71: 415e424.

18. Link TM, Lang T. Axial QCT: Clinical Applications and New Developments. J Clin Densitom 2014; 17: 438-448.

19. Link TM, Koppers BB, Licht T, et al. In vitro and in vivo spiral CT to determine bone mineral density: initial experience in patients at risk for osteoporosis. Radiology 2004; 231: 805e811.

20. Bauer JS, Henning TD, Mueller D, et al. Volumetric quantitative $\mathrm{CT}$ of the spine and hip derived from contrastenhanced MDCT: conversion factors. AJR Am J Roentgenol 2007; 188: $1294 \mathrm{e} 1301$.

21. Keyak JH, Sigurdsson S, Karlsdottir GS, et al. Effect of finite element model loading condition on fracture risk assessment in men and women: the AGES-Reykjavik study. Bone 2013; 57: $18 \mathrm{e} 29$.

22. ACR-SPR-SSR practice parameter for the performance of quatitative computed tomography (QCT) bone densitometry 2014 (Resolution 39). http://www.acr.org/ /media/DE78D218C7A64526A821A9E8645AB46D.pdf

23. Diederichs G, Engelken F, Marshall LM, et al. Diffuse idiopathic skeletal hyperostosis (DISH): relation to vertebral fractures and bone density. Osteoporos Int 2011; 22: 1789e1797. 